\title{
Ion Excitation in a Linear Quadrupole Ion Trap with an Added Octopole Field
}

\author{
A. L. Michaud ${ }^{*}$ A. J. Frank, ${ }^{\dagger}$ C. Ding, ${ }^{\ddagger}$ XianZhen Zhao, \\ and D. J. Douglas \\ Department of Chemistry, University of British Columbia, Vancouver, British Columbia, Canada
}

\begin{abstract}
Modeling of ion motion and experimental investigations of ion excitation in a linear quadrupole trap with a $4 \%$ added octopole field are described. The results are compared with those obtained with a conventional round rod set. Motion in the effective potential of the rod set can explain many of the observed phenomena. The frequencies of ion oscillation in the $x$ and $y$ directions shift with amplitude in opposite directions as the amplitudes of oscillation increase. Excitation profiles for ion fragmentation become asymmetric and in some cases show bistable behavior where the amplitude of oscillation suddenly jumps between high and low values with very small changes in excitation frequency. Experiments show these effects. Ions are injected into a linear trap, stored, isolated, excited for MS/MS, and then mass analyzed in a time-of-flight mass analyzer. Frequency shifts between the $x$ and $y$ motions are observed, and in some cases asymmetric excitation profiles and bistable behavior are observed. Higher MS/MS efficiencies are expected when an octopole field is added. MS/MS efficiencies $\left(\mathrm{N}_{2}\right.$ collision gas) have been measured for a conventional quadrupole rod set and a linear ion trap with a $4 \%$ added octopole field. Efficiencies are chemical compound dependent, but when an octopole field is added, efficiencies can be substantially higher than with a conventional rod set, particularly at pressures of $1.4 \times 10^{-4}$ torr or less. (J Am Soc Mass Spectrom 2005, 16, 835-849) @ 2005 American Society for Mass Spectrometry
\end{abstract}

$\mathrm{T}$ Three-dimensional (3-D) ion traps can benefit from geometries that distort the field from that of an ideal quadrupole $[1,2]$. The distortions are described mathematically by the addition of higher multipoles to the electric potential [1c, 1d, 2]. The most widely discussed distortion is the "stretched" ion trap [2], which has the end cap electrodes moved out so that the distance to the end cap, $z_{0}$, is increased over that of an ideal field, $z_{0}=r_{0} / \sqrt{2}$, where $r_{0}$ is the distance from the center to the ring electrode. It has been argued that the addition of higher order multipole fields of the correct sign to 3-D traps improves MS/MS efficiency [1c, 1f, 2], and allows faster ejection at the stability boundary $[2,3]$, to give higher scan speeds and improved mass resolution.

There is increasing interest in using linear quadrupoles as ion traps, both as stand alone mass analyzers with radial [4] or axial [5] ejection, or in combination with other mass analyzers (for a recent review see [6]).

Published online May 3, 2005

Address reprint requests to Dr. D. J. Douglas, Department of Chemistry, University of British Columbia, 2036 Main Mall, Vancouver, BC V6T 1Z1, Canada. E-mail: douglas@chem.ubc.ca

* Current address: Department of Geochemistry, Keck Facility, Arizona State University, Tempe, AZ 85287, USA.

+ Current address: Battelle Memorial Institute, 505 King Avenue, Columbus, $\mathrm{OH}$ 43201, USA.

$\ddagger$ Current address: Department of Chemistry, Fudan University, 220 Handan Rd., Shanghai 200433, China.
There is also interest in trapping and exciting ions for MS/MS at the relatively low pressures typical for operation of the last mass analyzing quadrupole in triple quadrupole systems, ca. $3 \times 10^{-5}$ torr [7]. Addition of higher multipoles to a linear ion trap might be expected to provide benefits similar to those seen with 3-D traps. Douglas and coworkers [8] have shown that an octopole field can be added to a linear quadrupole by using rod sets with rods equally spaced from the central axis but with one pair of rods different in diameter than the other pair, as shown in Figure 1. The electric potential within this rod set is given to a good approximation by

$$
\begin{aligned}
V(x, y)= & {\left[A_{0}+A_{2}\left(\frac{x^{2}-y^{2}}{r_{0}^{2}}\right)\right.} \\
& \left.+A_{4}\left(\frac{x^{4}-6 x^{2} y^{2}+y^{4}}{r_{0}^{4}}\right)\right]\left(U-V_{r f} \cos \Omega t\right)
\end{aligned}
$$

where $x$ is the distance from the center towards a smaller rod, $y$ is the distance from the center towards a larger rod, $r_{0}$ is the distance from the center to any rod, and $U$ and $V_{r f}$ are the amplitudes of dc and radio frequency (rf) voltages applied from pole to ground. In eq $1, A_{0}, A_{2}$, and $A_{4}$ are the dimensionless amplitudes of a constant potential (independent of position), the quadrupole potential and the octopole potential, re- 


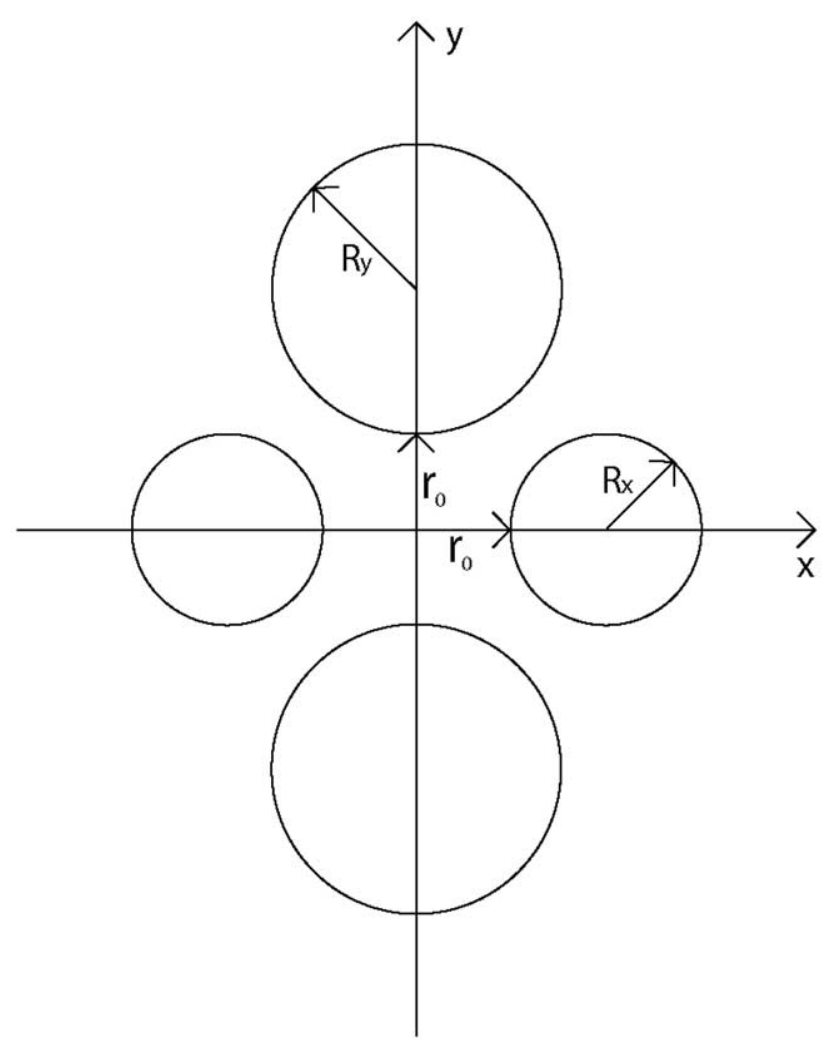

Figure 1. Cross sections of the electrodes of a linear quadrupole with added octopole field. The circular electrodes in the $x$ direction have radius $R_{x}$ and the circular electrodes in the $y$ direction radius $R_{y}$. All four electrodes are equally spaced from the central axis by a distance $r_{0}$. The rod set used here had $r_{0}=4.50 \mathrm{~mm}, R_{x}$ $=4.5 \mathrm{~mm}$, and $R_{y} / R_{x}=1.516$. The resulting multipole amplitudes are in Table 1.

spectively. The values of $A_{0}$ and $A_{4}$ are determined by the ratio $R_{y} / R_{x}$.

As with 3-D traps, the addition of an octopole field to a linear quadrupole field causes a variety of new effects. In this paper, we describe modeling of ion motion and experimental investigations of ion excitation in a linear quadrupole trap with a $4 \%$ added octopole field $\left(A_{4}=\right.$ $0.0398)$. The results are compared to those obtained with a conventional rod set with $A_{4}=0$. We derive the effective- or pseudo-potential $[9,10]$ arising from the potential of eq 1. Motion of an ion in the effective potential with collisions and excitation is that of a forced, damped, anharmonic oscillator. The frequencies of ion oscillation in the $x$ and $y$ directions shift with increasing amplitude in equal but opposite directions. Excitation profiles for ion ejection or fragmentation become asymmetric and in some cases can show bistable behavior where the amplitude of oscillation suddenly jumps between high and low values. These sudden jumps in amplitude can in principle be used to produce high resolution in ion isolation or ion ejection. Experiments show many of these effects. Ions are injected into a linear trap, stored, isolated, excited for MS/MS, and then mass analyzed in a linear time-offlight mass analyzer [11, 12]. Frequency shifts between the $x$ and $y$ motions are observed, and in some cases asymmetric excitation profiles and bistable behavior are observed. Higher MS/MS efficiencies are expected when an octopole field is added. We have measured MS/MS efficiencies ( $\mathrm{N}_{2}$ collision gas) with a conventional quadrupole rod set and a linear ion trap with $A_{4}$ $=0.0398$. Efficiencies are chemical compound dependent, but when an octopole field is added, they can be substantially higher than with a conventional rod set, particularly at pressures of $1.4 \times 10^{-4}$ torr or less.

\section{Experimental}

A schematic of the system used, similar to a trap-TOF system described previously [11,12], is shown in Figure 2. Ions formed by electrospray pass through a curtain gas (dry $\mathrm{N}_{2}, 99.999 \%$ stated purity, Praxair Canada Inc., Delta, British Columbia, Canada), and enter the vacuum system through a $0.25 \mathrm{~mm}$ diameter orifice and then a $0.75 \mathrm{~mm}$ diameter skimmer orifice in a differentially pumped region at a background pressure of 1.0 torr. Ions then enter a quadrupole ion guide $\mathrm{Q} 0,30 \mathrm{~cm}$ long with a field radius of $4.5 \mathrm{~mm}$, pumped to a pressure of about $3.5 \mathrm{mTorr}$ by a $230 \mathrm{~L} / \mathrm{s}$ turbomolecular pump (TPH 240, Balzers, Nashua, $\mathrm{NH}$ ). Ions then pass through a $2 \mathrm{~mm}$ diameter aperture, the entrance lens, into a linear quadrupole ion trap Q1, pumped to a base pressure of $3 \times 10^{-6}$ torr by a $550 \mathrm{~L} / \mathrm{s}$ turbomolecular pump (TV 551, Varian, Torino, Italy). The pressure in the trap could be increased above the base pressure by adding nitrogen $(99.999 \%$ stated purity, Praxair Canada Inc.) to the chamber through a needle valve. The trap pressure was measured with an ion gauge for pressures below $1 \times 10^{-4}$ torr and a 0.1 torr capacitance manometer for pressures above $1 \times 10^{-4}$ torr. Both were calibrated against a high precision capacitance manometer (Baratron model 120, manufacturer's stated accuracy $0.12 \%$ of reading, MKS, Boulder, CO). Two traps, Q1, each $20 \mathrm{~cm}$ long were used: a conventional quadrupole rod set with round rods field radius $4.17 \mathrm{~mm}$, and ratio of rod radius to field radius $\mathrm{R} / \mathrm{r}_{0}=1.126$, or a quadrupole rod set with a nominal $4 \%$ octopole field $\left(A_{4}=0.0398\right)$ (the largest $A_{4}$ of the rod sets with added octopole fields that we have constructed) and field radius of $4.5 \mathrm{~mm}$ (Figure 1) [8]. The dimensionless amplitudes of the higher order multipoles in these rod sets are shown in Table 1. Ions were confined in Q1 by applying trapping potentials to the entrance and exit apertures. Ions then passed through a $2 \mathrm{~mm}$ diameter aperture in a cone and three aperture lenses (L1-L3), 6.4 $\mathrm{mm}$ i.d. (spacing $2.5 \mathrm{~mm}$ ) into the source region of a linear time-of-flight (TOF) mass analyzer (R. M. Jordan Co., Grass Valley, CA). The TOF, with a mass resolution limited to several hundred [11], is pumped by a $360 \mathrm{~L} / \mathrm{s}$ turbomolecular pump (Turbovac 361, Leybold, Mississauga, Ontario, Canada) to a base pressure of $3 \times 10^{-7}$ torr.

Isolation waveforms, notched in frequency space, were created with SxWave software (Pan Galactic Scientific, Peterborough, Ontario, Canada) using a "comb" of frequencies separated by $250 \mathrm{~Hz}$ with random 


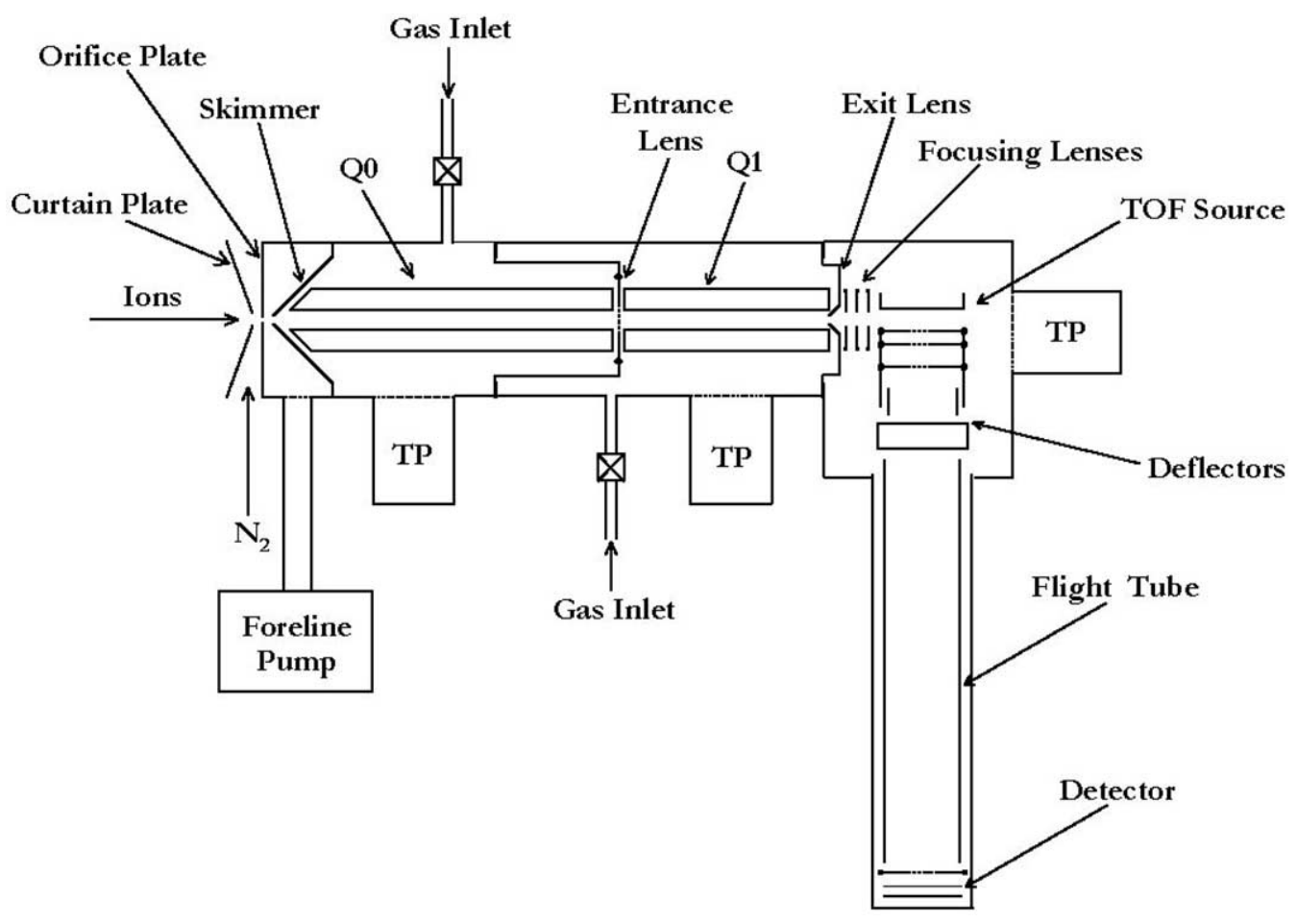

Figure 2. The linear ion trap time-of-flight system used for these experiments.

phases. The isolation window notch was $6 \mathrm{kHz}$, centered at the calculated secular frequency of the ion of interest for an ideal quadrupole rod set. The resulting list of amplitudes was then downloaded via an RS232 interface to an arbitrary waveform generator, (model 33120A, Agilent Technologies, Palo Alto, CA). Isolation times were $20 \mathrm{~ms}$ and amplitudes were chosen to minimize the background signal with minimal loss of the precursor. We argue below that the rod sets with added octopole fields favor ion fragmentation over ion ejection. Nevertheless, no difficulty was found in isolating ions. With a sufficiently large excitation amplitude $\left(4.0 \mathrm{~V}_{0-p}\right)$, ions could still be efficiently ejected from the trap with added multipole field [1a]. Dipole excitation waveforms were generated with an arbitrary waveform generator (SRS DS345, Stanford Research Systems, Sunnyvale, CA). Excitation voltages in this paper are reported as volts zero to peak, pole to ground $\left(\mathrm{V}_{0-p}\right)$. In a pure quadrupole field the angular frequencies of ion oscillation are given by

$$
\omega_{u}=\left(2 n+\beta_{u}\right) \frac{\Omega}{2}
$$

where $\mathrm{n}=0, \pm 1, \pm 2 \ldots, u=x$ or $y, \beta$ is a function of the trapping voltage, and $\Omega$ is the angular frequency of the trapping $\mathrm{rf}$ voltage $(\Omega=2 \pi 768.144 \mathrm{kHz}$; generated by an API 3 quadrupole power supply [SCIEX, Concord, Ontario, Canada]). The fundamental frequencies with $\mathrm{n}=0$ were excited.

Sprayer voltages were ca. $4.3 \mathrm{kV}$. The curtain plate was held at $1.1 \mathrm{kV}$ and the orifice was maintained at ca. $180 \mathrm{~V}$, except when fragmenting ions of morphine-3- $\beta$ D-glucuronide in the orifice-skimmer region, when the orifice voltage was increased to ca. $240 \mathrm{~V}$. The skimmer was usually held in the range of 5 to $12 \mathrm{~V}$ and the ion guide rod offset was 2 to $5 \mathrm{~V}$. The trap entrance aperture plate was at $-5 \mathrm{~V}$ to $+0.5 \mathrm{~V}$ and there was no DC offset for the trap. The exit lens was typically at $-5 \mathrm{~V}$. To block ions, the potentials of the entrance or exit lens were set $25 \mathrm{~V}$ higher than the lower voltage settings. The lens stack was operated with L1, L2, and L3 at -25 $\mathrm{V},-22 \mathrm{~V}$, and $-215 \mathrm{~V}$, respectively. The TOF source pulsing was controlled by a pulse delay generator (BNC 555, Berkeley Nucleonics, San Rafael, CA). This consisted of 20 pulses (width $30 \mu \mathrm{s}$ ) at $380 \mathrm{~V}$ and a frequency of $3.333 \mathrm{KHz}$ every time the trap was emptied. Pulsed ions passed through vertical and horizontal deflectors held at $-2.5 \mathrm{kV}$ and $-2.2 \mathrm{kV}$, respectively, and into the flight tube, maintained at $-2.6 \mathrm{kV}$. Two 25 $\mathrm{mm}$ active area microchannel plates (Burle Electro-

Table 1. Multipole amplitudes

\begin{tabular}{lrlllll}
\hline Rod set & $\mathrm{A}_{0}$ & \multicolumn{1}{c}{$\mathrm{A}_{2}$} & $\mathrm{~A}_{4}$ & \multicolumn{1}{c}{$\mathrm{A}_{6}$} & $\mathrm{~A}_{8}$ & $\mathrm{~A}_{10}$ \\
\hline \hline Conventional & 0.0000 & 1.00149 & 0.0000 & 0.001267 & 0.0000 & -0.002431 \\
4\% Octopole & -0.0403 & 1.0056 & 0.03980 & -0.00299 & 0.0008702 & -0.002319 \\
\hline
\end{tabular}


optics Inc., Sturbridge, MA) in a chevron configuration were used to detect ions.

Timing was controlled using the software package Benchtop Lite (PC Instruments Inc., Lawrence, KS) running on a Pentium III desktop PC. For MS/MS, six timing segments were set: emptying, injection, isolation, excitation, cooling, and detection. The periods for trap emptying and injection were $40 \mathrm{~ms}$, for isolation 20 $\mathrm{ms}$, for excitation from $50 \mathrm{~ms}$ to about $500 \mathrm{~ms}$, and for detection $10 \mathrm{~ms}$. The cooling period was varied according to the collision gas pressure. Typical cooling times were $10 \mathrm{~ms}, 20 \mathrm{~ms}, 100 \mathrm{~ms}$, and $500 \mathrm{~ms}$ for pressures ranging from $10^{-3}$ to $10^{-6}$ torr, respectively. These periods were selected such that little increase in the precursor ion signal was observed for longer cooling periods. Spectra were acquired with a multichannel scalar (Turbo-MCS, Ortec International Inc., Atlanta, GA) and the associated MCS-32 software package running on a $133 \mathrm{MHz}$ desktop PC. Dwell times were typically $40 \mathrm{~ns}$.

HPLC grade acetonitrile, and HPLC grade methanol were from Aldrich (Sigma-Aldrich, Oakville, Ontario, Canada). Morphine-3- $\beta$-D-glucuronide, as a stock analytical solution of $2.0 \mathrm{mg} / \mathrm{ml}$ in methanol, and reserpine, were from Sigma (Sigma-Aldrich). Sample concentrations were typically $50 \mu \mathrm{M}$, infused to the ESI source at $1 \mu \mathrm{L} / \mathrm{min}$. Reserpine was dissolved in pure acetonitrile. Morphine-3- $\beta$-D-glucuronide was diluted from the stock solution into 1:1 water:methanol with $0.5 \% \mathrm{vol} / \mathrm{vol}$ acetic acid.

Ion trajectories were calculated with a program written by Michael Sudakov (unpublished). Following input of all the data, such as rf frequency, Mathieu parameter $q$, collision gas mass, pressure and temperature, collision cross section, excitation frequency and amplitude, octopole amplitude $A_{4}$, and initial $x$ and $y$ positions and velocities, the equations of motion are integrated to produce values of $x$ and $y$ versus time. Collisions are included in the trajectories approximately by using a drag coefficient model as described below. An ideal quadrupole field or a quadrupole field with added octopole component could be modeled. Higher spatial harmonics that result from using round rod sets were not included in the trajectory calculations.

\section{Results and Discussion}

\section{Effective Potential and Frequency Shifts}

Many aspects of ion motion in the linear trap with an added octopole field can be understood by considering the effective potential for the time-dependent potential given by eq 1 with $U=0$. For an ion of mass, $m$, and charge, $e$, in an inhomogeneous electric field, $\vec{E}$, oscillating at angular frequency $\Omega$, the effective electric potential [10] is given by

$$
V_{e f f}=\frac{e|\vec{E}|^{2}}{4 m \Omega^{2}}
$$

where

$$
|\vec{E}|^{2}=\left(E_{x}^{2}+E_{y}^{2}+E_{z}^{2}\right)
$$

In eq 3 and 4 only the spatial dependence of the potential is included. For the potential of eq 1 , eq 3 leads to

$$
V_{\text {eff }}=\frac{q A_{2}^{2} V_{r f}}{4}\left(\frac{x^{2}+y^{2}}{r_{0}^{2}}\right)+\frac{q A_{2} A_{4} V_{r f}}{1}\left(\frac{x^{4}-y^{4}}{r_{0}^{4}}\right)+\cdots
$$

where the Mathieu parameter $q$ is given by

$$
q=\frac{4 e V_{r f}}{m r_{0}^{2} \Omega^{2}}
$$

The first term in eq 5 describes the well known effective potential for a linear rf-only quadrupole, and the second term describes the modifications caused by the octopole field. Terms in $x^{n} y^{m}$ have not been included because we consider here excitation of the $x$ motion when $y=0$ and excitation of the $y$ motion when $x=0$. In the direction of the $x$ rods the effective potential increases more rapidly with distance from the center than that of a pure quadrupole potential, and in the $y$ direction increases less rapidly than that of a quadrupole potential. Thus the effects of positive and negative octopole components can be studied in the same rod set by considering the $x$ and $y$ motions, respectively.

Motion of a singly charged ion in $x$ direction in the effective potential of eq 5 is described by

$$
m \ddot{x}=F_{x}=-e \frac{\partial V_{e f f}}{\partial x}=-\frac{e q A_{2}^{2} V_{r f} 2 x}{4 r_{0}^{2}}-\frac{4 e q A_{2} A_{4} V_{r f} x^{3}}{r_{0}^{4}}
$$

where $\ddot{x}=\frac{d^{2} x}{d t^{2}}$. Eq 7 can be written

$$
\ddot{x}+\omega_{0}^{2} x=-\frac{4 e q A_{2} A_{4} V_{r f} x^{3}}{m r_{0}^{4}}
$$

with

$$
\omega_{0}^{2}=\frac{e q A_{2}^{2} V_{r f^{2}}}{4 m r_{0}^{2}}
$$

which can be rewritten as

$$
\omega_{0}=\frac{q A_{2} \Omega}{\sqrt{8}}
$$


Eq 10 is the well known expression for the secular frequency of an ion in a quadrupole field at low $q$ values [13]. The octopole field adds the term in $x^{3}$ to the right of eq 8 .

Landau and Lifshitz [9] have considered motion of an anharmonic oscillator described by

$$
\ddot{x}+\omega_{0}^{2} x=-\alpha x^{2}-\beta x^{3}
$$

Eq 11 is the same as eq 8 when $\alpha=0$ and $\beta=$ $\frac{4 e q A_{2} A_{4} V_{r f}}{m r_{0}^{4}}$. ( The term $-a x^{2}$ on the right of eq 11 can be contributed to the effective potential when a hexapole field is added to the potential of eq 1). The anharmonic terms on the right of eq 11 cause shifts in the resonant frequency away from $\omega_{0}$, given [9] by

$$
\Delta \omega=\left(\frac{3 \beta}{8 \omega_{0}}-\frac{5 \alpha^{2}}{12 \omega_{0}^{3}}\right) b^{2}
$$

where $b$ is the amplitude of oscillation. Thus the octopole field causes a frequency shift given by

$$
\Delta \omega=\frac{3 e q A_{2} A_{4} V_{r f}}{2 m r_{0}^{4} \omega_{0}} b^{2}
$$

Substituting for $V_{r f}$ from eq 9 gives

$$
\Delta \omega=3 \frac{A_{4}}{A_{2}}\left(\frac{b^{2}}{r_{0}^{2}}\right) \omega_{0}
$$

The same result was derived by Sevugarajan and Menon [14] for $z$ motion in the effective potential of a 3-D ion trap with an added octopole field; however, in the 3-D trap, the unperturbed frequencies in the $\mathrm{z}$ and $\mathrm{r}$ directions differ by a factor of two. Although eq 14 was derived from the effective potential approximation, which is normally considered valid for $q<0.4$, direct simulations of ion motion in a stretched 3-D trap show the frequency shift is independent of $q$ within about $10 \%$ for $q$ up to 0.8 [15]. When $A_{4}>0$ ( $x$ motion) there is a shift to higher frequency which is proportional to $A_{4}$ and to the square of the amplitude of ion oscillation. For $A_{4}<0$ (y motion) there is a shift to lower frequency of the same magnitude for the same amplitude of oscillation.

\section{Axis Potential}

From eq 1, these rod sets have an axis potential $A_{0}\left(U-V_{r f} \cos \Omega t\right)$ where $A_{0} \approx-A_{4}$ [8a]. The axis potential does not affect ion motion within the linear trap because it is independent of position and therefore produces no electric fields. However, it can affect introduction of ions or draining of ions through the fringing fields at the ends of the trap. The axis potential can be removed by applying a greater voltage to the smaller rods and a lower voltage to the larger rods. When the rf was unbalanced in this way to make the axis potential zero, the signal observed in an experiment where ions were trapped and then allowed to drain into the TOF mass analyzer increased by $20 \%$. Thus the axis potential does not have a large effect on the efficiency of ion injection or extraction from the trap. The experiments described here were done with balanced $\mathrm{rf}$ applied to the rods.

\section{Experimental Frequency Shifts and Peak Shapes}

Figure 3 shows the results of an experiment to measure the frequencies and peak shapes for depletion of protonated reserpine ions and formation of fragment ions with dipole excitation applied between the larger (Figure $3 a$ and $b$ ) and smaller (Figure $3 c$ and $d$ ) rods. Ions were excited at a pressure of $2.0 \mathrm{mTorr}$ of $\mathrm{N}_{2}$ for $100 \mathrm{~ms}$ with an amplitude of $154 \mathrm{mV}_{0-p}$. After excitation, ions were transferred to the source region of the TOF for mass analysis and the intensities of the reserpine ions and fragments were measured. It is immediately apparent that the resonances for the two directions have different frequencies. The calculated unperturbed frequency of motion, $\frac{\omega_{0}}{2 \pi}$, for $q=0.2024(\beta=0.1443)$ is $55.44 \mathrm{kHz}$. The resonance for excitation between the smaller rods, which have a positive $A_{4}$ in the effective potential, shows a shift up from $55.44 \mathrm{kHz}$ of $1.11 \mathrm{kHz}$ to $56.55 \mathrm{kHz}$, and for excitation between the larger rods a shift down of $1.24 \mathrm{kHz}$ to $54.20 \mathrm{kHz}$. These shifts are approximately equal in magnitude and of opposite sign as expected from eq 14. Small differences in the shifts can be caused by nonlinearities in the electronics and by higher multipoles in the potential. For a given applied voltage, excitation between the larger rods produces a slightly higher dipole field than excitation between the smaller rods (see below), and this may contribute to the slightly greater shift with the larger rods.

The depletion of reserpine ions in Figure 3 is not directly related to the amplitude of oscillation in any simple way, since it corresponds to removal of ions by dissociation following collisional activation [12]. Nevertheless, from eq 14, frequency shifts of $1.11 \mathrm{kHz}$ and 1.24 $\mathrm{kHz}$ correspond to amplitudes of oscillation $b=0.41 r_{0}$ and $b=0.43 r_{0}$, respectively. These amplitudes, and the formation of fragment ions shown in Figure $3 b$ and $d$, indicate that the ions are lost by dissociation, not ejection.

The peaks shown in Figure $3 a$ and $b$ are asymmetric, with a sharp side at lower frequencies and a tail to higher frequencies. In contrast, the peaks in Figure 3c and $\mathrm{d}$ are considerably more symmetric. An asymmetric peak is expected from motion in the effective potential given by eq 5 . As described by Landau and Lifshitz [9], a plot of the amplitude of oscillation versus excitation frequency for forced oscillation of the oscillator described by eq 11 is no longer symmetric about the resonant frequency $\omega_{0}$. The forced anharmonic motion with damping is described by 

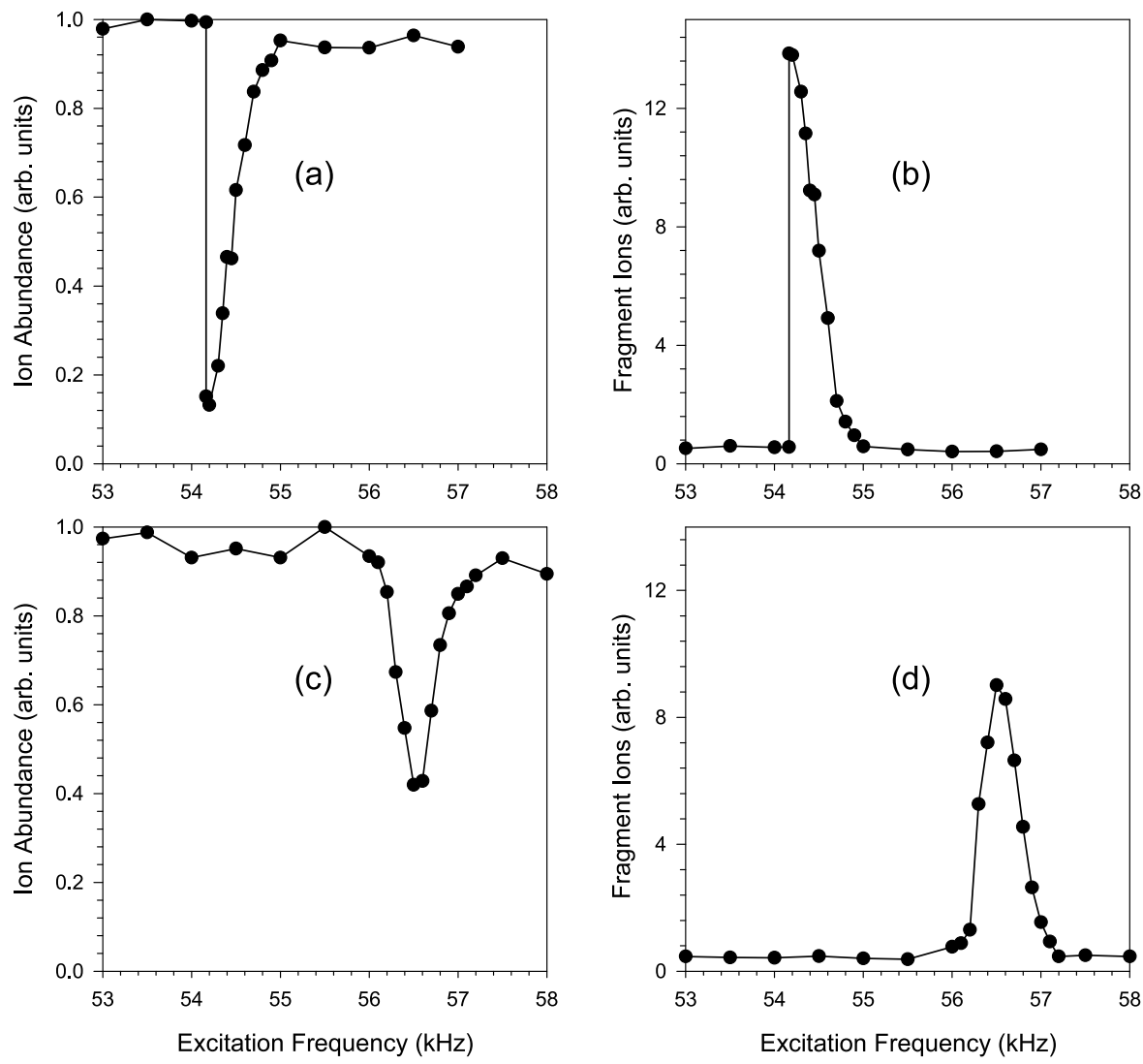

Figure 3. Experimental resonance peak shapes for removal of reserpine ions and formation of fragment ions with dipole excitation. Trapping rf frequency $768.144 \mathrm{kHz}, q=0.2021,2.0 \mathrm{mTorr}^{\text {of }} \mathrm{N}_{2}$, excitation amplitude $153 \mathrm{mV}_{0-p}$. (a) Precursor and (b) sum of fragment ion intensities with excitation between the larger rods (c) precursor and (d) sum of fragment ion intensities with excitation between the smaller rods.

$$
\ddot{x}+2 \lambda x+\omega_{0}^{2} x=(f / m) \cos \gamma t-\alpha x^{2}-\beta x^{3}
$$

where $\lambda$ is a damping constant and $f$ is the amplitude of the driving force with angular frequency $\gamma$. If $\beta$ is positive $\left(A_{4}>0\right.$, excitation between the smaller rods) then a plot of amplitude of oscillation versus frequency shows a sharper side on the high frequency sides of the peak. If $\beta$ is negative $\left(A_{4}<0\right.$, excitation between the larger rods) the sharper side is the low frequency side, as seen in Figure 3a. Asymmetric peaks have been seen in 3-D traps and attributed to field distortions, but the connection to specific multipoles, to the sign of the multipoles, or to effective potentials has received little discussion. March et al. [16] attributed frequency shifts in a 3-D trap to higher order components of the field, but did not discuss these shifts in terms of multipoles. Williams et al. [17] observed asymmetric peaks with a tail to the low frequency side for excitation of ions of butylbenzene in a stretched 3-D trap, although again the results were not discussed in terms of multipoles of the potential. In an ion trajectory simulation of motion in a stretched trap, Splendore et al. [18] found a frequency shift approximately proportional to the square of the amplitude of ion motion, with a shift to higher frequency for $z$ motion and to lower frequencies for $r$ motion, as expected qualitatively from eq 14 . If the fre- quency shift observed by Splendore et al. is attributed to the presence of an octopole with no other higher multipoles, an octopole component of ca. 1\% can be calculated from eq 14. Alheit et al. [19a] found an asymmetric peak with a tail to the high frequency side, for quadrupole excitation of the collective motion of ions in a 3-D trap with ideal geometry. The asymmetry was attributed to an added hexapole field, which causes the frequency of oscillation to decrease as the amplitude increases. The same group reported asymmetric peaks for excitation of higher order resonances $(n>0$ in eq 2$)$. For the higher order resonances the effective potential approximation no longer applies and it was found peaks could have tails on either the low or high frequency sides [19b]. Nappi et al. [20] observed shifts to lower frequency for $z$ motion in a stretched trap; the shifts were attributed to addition of $1.4 \%$ octopole and $3 \%$ hexapole to the $3-\mathrm{D}$ field, although the frequency shifts caused by these added fields were not calculated.

\section{Bistable Behavior}

The low frequency sides of the peaks in Figure $3 a$ and $b$ are remarkably sharp. Increasing the excitation frequency by $0.001 \mathrm{~Hz}$ from $54.163845 \mathrm{kHz}$ to 54.163846 


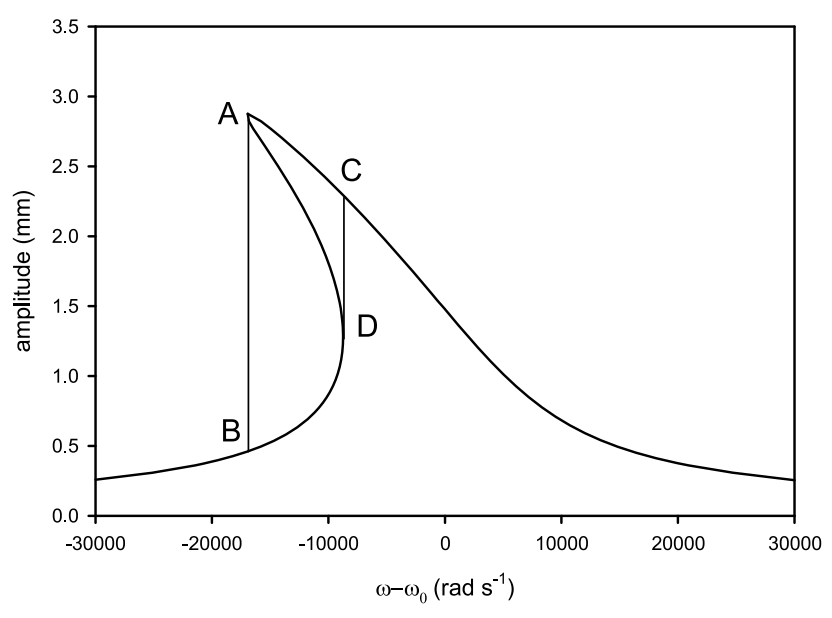

Figure 4. Amplitudes of oscillation for reserpine ions $\mathrm{m} / \mathrm{z}=609$ trapped in a quadrupole field with $4 \%$ added octopole field versus shift in the angular excitation frequency from the unperturbed frequency. Trapping rf frequency $768 \mathrm{kHz}, q=0.2026,2.0$ mTorr of $\mathrm{N}_{2}$, excitation voltage $189 \mathrm{mV}_{0-p}$, applied between the larger rods.

$\mathrm{kHz}$ causes a sudden jump in depletion of the precursor with a sudden increase in the formation of fragment ions. This sudden jump is likely the result of bistable behavior, expected for a nonlinear oscillator, and caused by the anharmonic terms on the right of eq 15 [9, $21,22]$. Bistable behavior has been observed in 3-D traps by Alheit et al, [19a] and Chu et al. [19b], and attributed to anharmonicities in the potential. In their work, a sharp boundary on the low frequency side of the resonance was attributed to the presence of an added hexapole potential.

As the driving force in eq 15 increases, a plot of the amplitude of oscillation versus driving frequency becomes increasingly asymmetric, and at a critical force, $f_{c}$, bistable behavior is formed [9]. With a negative octopole potential, the form of the curve of amplitude of oscillation versus excitation frequency takes on the appearance of Figure 4. The amplitude of oscillation, $b$, is related to the driving frequency through

$$
\epsilon=\kappa b^{2} \pm \sqrt{\left[\left(\frac{f}{2 m \omega_{0} b}\right)^{2}-\lambda^{2}\right]}
$$

where $\epsilon=\gamma-\omega_{0}$ [9].

The critical force is given by

$$
f_{c}^{2}=\frac{32 m^{2} \omega_{0}^{2} \lambda^{3}}{3 \sqrt{3} \kappa}
$$

where $\kappa$ is related to the frequency shift by $\omega=\omega_{0}+$ $\kappa b^{2}$.

From eq 14

$$
\kappa=3 \frac{A_{4}}{A_{2}}\left(\frac{1}{r_{0}^{2}}\right) \omega_{0}
$$

For example in our rod set with $A_{4} / A_{2}=0.04, r_{0}=4.5$ $\times 10^{-3} \mathrm{~m}$, and $\omega_{0} \approx 2 \pi 5.5 \times 10^{4} \mathrm{~s}^{-1}, \kappa=2.05 \times 10^{9} \mathrm{~s}^{-1}$ $\mathrm{m}^{-2}$.

The damping constant, $\lambda$, can be related to the collision cross section and gas number density with a drag coefficient model [23] which gives the force, $F_{d}$, on an ion as

$$
F_{d}=-\frac{C_{d} \sigma n m_{2} v^{2}}{2}
$$

where $C_{d}$ is a drag coefficient, $\sigma$ the collision cross section, $n$ the gas number density, $m_{2}$ the collision gas mass, and $v$ the ion speed. If the drag force on a particle is $F_{d}=-\alpha^{\prime} v$ Landau and Lifshitz [9] define a damping constant by

$$
\frac{\alpha^{\prime}}{m}=2 \lambda
$$

For low ion speeds,

$$
C_{d}=\frac{3.01 \sqrt{2 k T / m_{2}}}{v}
$$

where $T$ is the gas temperature and $m_{2}$ is the collision gas mass [23]. Thus

$$
F_{d}=\frac{-3.01 \sqrt{2 k T / m_{2}} \sigma n m_{2} v}{2}
$$

so that

$$
\alpha^{\prime}=\frac{3.01 \sqrt{2 k T / m_{2}} \sigma n m_{2}}{2}
$$

and

$$
\lambda=\frac{\alpha^{\prime}}{2 m}=\frac{3.01 \sqrt{2 k T / m_{2}} \sigma n m_{2}}{4 m}
$$

For ions of reserpine $\left(\sigma=280 \AA^{2}\right.$ [24] ) trapped at $2.0 \times$ $10^{-3}$ torr of $\mathrm{N}_{2}$ at $295 \mathrm{~K}, \lambda=2.67 \times 10^{3} \mathrm{~s}^{-1}$. Thus the critical force for reserpine ions under these conditions is $f_{c}=2.65 \times 10^{-18} \mathrm{~N}$. The force is related to the electric field, $E$, by $f=e E$, so the electric field that produces this critical force on a reserpine ion is calculated to be $16.6 \mathrm{~V}$ $\mathrm{m}^{-1}$.

The electric field with dipole excitation can be calculated for the rod geometry of Figure 1 by the method of equivalent charges [25]. The dipole potential within the rods is given by

$$
\phi_{1}=A_{1}\left(\frac{u}{r_{0}}\right) V \cos \omega t
$$

where $u$ is $x$ or $y$ and the electric field is 
Table 2. Amplitudes of the dipole field for different rod sets and rod pairs

\begin{tabular}{llc}
\hline Ratio of rod radii & Excitation & $\mathrm{A}_{1}$ \\
\hline \hline 1.516 & Small rods & 0.775 \\
1.516 & Large rods & 0.826 \\
1.000 & Either rod pair & 0.798 \\
\hline
\end{tabular}

$$
E=-A_{1}\left(\frac{V}{r_{0}}\right) \cos \omega t
$$

The amplitudes of the dipole field, $A_{1}$, produced in the rod sets used in these experiments were calculated with the results shown in Table 2 . Thus, to produce an electric field with amplitude $16.6 \mathrm{~V} \mathrm{~m}^{-1}$ requires an excitation voltage between the large rods of $V=\frac{16.6}{0.826} 4.5 \times 10^{-3}=$ $0.090 \mathrm{~V}$ zero to peak, pole to ground. The data of Figure 4 were calculated from eq 16 using these values of $\kappa, \lambda$, and eq 26 to convert the force from the $189 \mathrm{mV}_{0-p}$ voltage to a force on an ion of $5.6 \times 10^{-18} \mathrm{~N}$. The data of Figure 3 (excitation amplitude $153 \mathrm{mV}_{0-p}$ ) and the curve of Figure 4 (excitation amplitude $189 \mathrm{mV}_{0-p}$ ) both have excitation amplitudes above the amplitude necessary to produce the critical force.

Figure 4 shows that, starting at a low frequency, as the frequency is increased the amplitude of oscillation increases. However, beginning at the point $B$ there are three possible solutions for the amplitude. If the frequency of excitation is increased, ions can follow the curve to $D$, then suddenly jump in amplitude to $C$. If the frequency of excitation is decreased, starting at the right of Figure 4, ions can follow the upper curve past $C$ to $A$ where the amplitude suddenly decreases. The use of this type of behavior to improve scan speed and ion ejection from a 3-D trap has been described by Makarov [22].

When a positive octopole component is present, as with excitation between the smaller rods, the curve also has bistable behavior but the curve is reflected about the $\omega-\omega_{0}=0$ axis. Thus we would expect to see a sharp side on the high frequency side of the peak. However the experiments, Figure $3 \mathrm{c}$ and $\mathrm{d}$ show fairly symmetric peaks. The data of Figure 4 are based on an approximate solution to eq 15, which is itself an approximation based on the effective potential. To more accurately determine the amplitudes of oscillation, trajectory calculations were used to determine the amplitude of oscillation for different excitation frequencies, with the results shown in Figure 5. The excitation amplitude, 250 $\mathrm{mV}_{0-p}$ is well above the critical amplitude of $90 \mathrm{mV}_{0-p}$. The curve " $a$ " is for excitation of ions between the smaller rods and the curve " $b$ " for excitation between the larger rods. The sudden change in amplitude with frequency can be seen clearly in each case. In the trajectory calculations, the difference in frequency between the point of highest amplitude and the next point of much lower amplitude is $1 \mathrm{~Hz}$. Similar excitation profiles, which showed a sharp transition on the high frequency side of the peak, were reported by Splendore et al. for simulations of ion motion in the $z$ direction of a stretched 3-D trap [18]. The results were not interpreted in terms of multipoles or anharmonic oscillations.

Despite these expectations from modeling the motion in the effective potential (Figure 4) and from direct trajectory calculations (Figure 5), the experiments (Figure $3 c$ and d) do not show a sharp side on the high frequency side of the peak. If sharp sides can be produced on both the low and the high frequency sides, then high mass resolution in ion isolation will be possible, as shown for a 3-D trap by Ding et al. [26]. In that work, positive or negative multipoles could be added to the field of a 3-D trap by changing the voltage on a field adjusting electrode. Scans up in mass and down in mass with resonant ejection could then be combined to give a resolution of greater than 3500, demonstrated with isolation of monoisotopic peaks of insulin $\beta$-chain +3 ions at $m / z \approx 1166$.

\section{MS/MS Efficiencies}

It has been argued that MS/MS efficiencies increase when higher multipoles are added to a 3-D trap [1c, 1f, 2]. The same improvements might be expected for a linear trap [7, 8a]. MS/MS efficiencies were measured for reserpine ions trapped in a conventional rod set and in the rod set with $4 \%$ octopole field. The effects of pressure, excitation amplitude, and excitation time were investigated. In all experiments, ions were trapped at $q \approx 0.20$ with excitation for $100 \mathrm{~ms}$, and the excitation amplitude was varied. Then the excitation was kept fixed at approximately the amplitude that gave $50 \%$ depletion of the precursor ion, and the excitation time was varied. (The data are not corrected

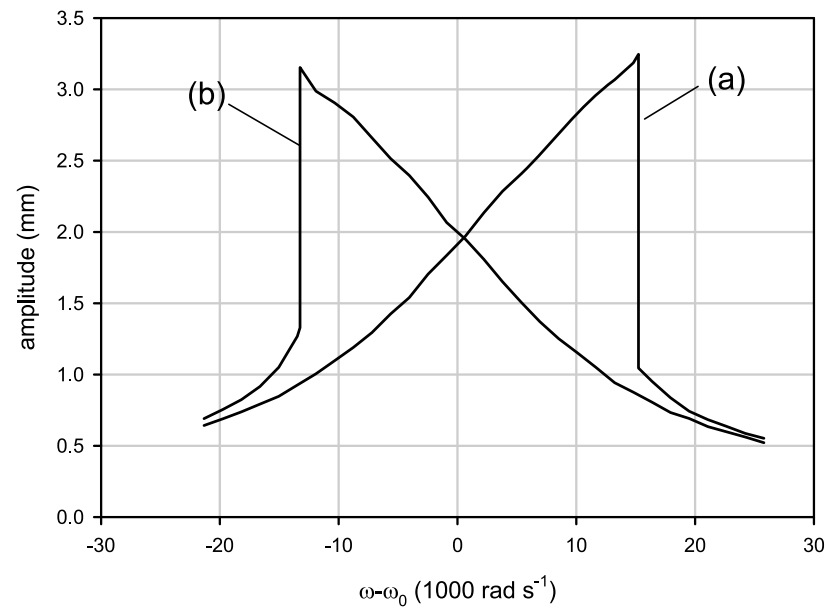

Figure 5. Results of trajectory calculations. Oscillation amplitude versus excitation frequency for excitation between (a) the smaller rods and (b) the larger rods. Ion $m / z=609, A_{4}=0.040, q=0.2026$, excitation voltage $0.25 \mathrm{~V}_{0-p}, 2.0 \mathrm{mTorr} \mathrm{N}_{2}, \sigma=280 \AA^{2}$, trapping rf frequency $768 \mathrm{kHz}, \mathrm{r}_{0}=4.5 \mathrm{~mm}$. Initial $x$ and $y$ positions $0.1 r_{0}$, initial radial velocities 0 . 

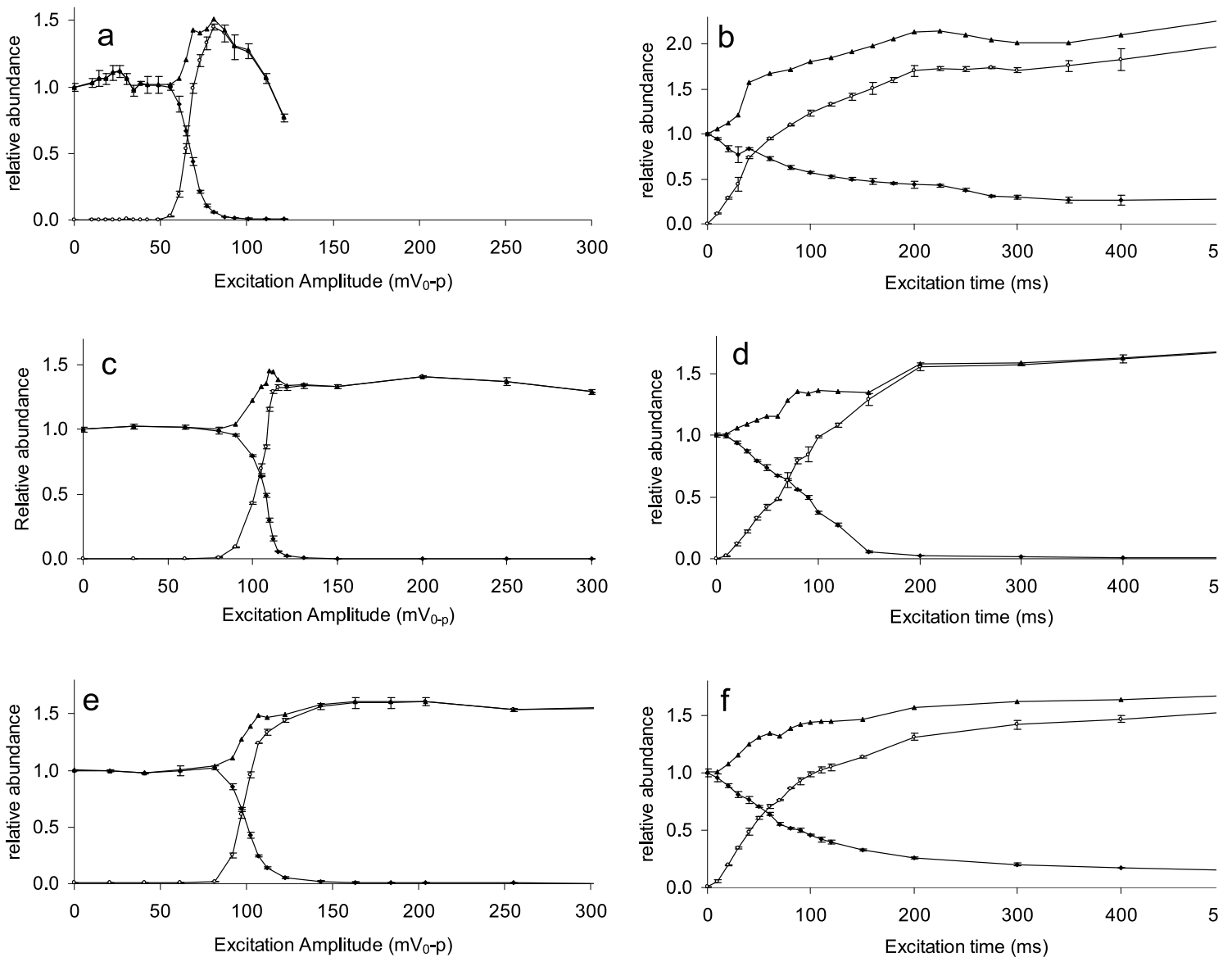

Figure 6. Ion intensities in MS/MS of reserpine ions at ca. $1.05 \mathrm{mTorr}$. In each plot the decrease of precursor ion, sum of all fragment ions, and sum of all ions in the spectrum are shown. (a) Conventional rod set, 1.08 mTorr, intensities versus excitation amplitude ( $54.2 \mathrm{kHz}),(\mathbf{b})$ conventional rod set, 1.08 mTorr, intensities versus excitation time, amplitude $69 \mathrm{mV}_{0-p}(54.2 \mathrm{kHz})$, (c) rod set with $A_{4}=0.04,1.15$ mTorr, excitation between the larger rods, intensities versus excitation amplitude (54.0 $\mathrm{kHz}$ ), (d) rod set with $A_{4}=0.04,1.15$ mTorr, excitation between the larger rods, intensities versus excitation time, amplitude $106 \mathrm{mV}_{0-p}(54.0 \mathrm{kHz})$, (e) rod set with $A_{4}=0.04,1.08 \mathrm{mTorr}$, excitation between the smaller rods, intensities versus excitation amplitude $(56.9 \mathrm{kHz}),(\mathbf{f}) \operatorname{rod}$ set with $A_{4}=0.04$, 1.08 mTorr, excitation between the smaller rods, intensities versus excitation time, amplitude $96 \mathrm{mV}_{0-p}$ $(56.9 \mathrm{kHz})$.

for $m / z$ dependent ion transmission into and through the TOF mass analyzer, and therefore the amplitude giving $50 \%$ depletion of the precursor ion is not the amplitude giving equal precursor and fragment ion intensities.) Results of experiments at trap pressures of about 1.05, 0.14, and 0.035 mTorr of $\mathrm{N}_{2}$ are shown in Figures 6, 7, and 8, respectively. Each plot in these figures shows the abundance of precursor ions, the sum of fragment ions and the sum of all ions.

Figure 6a shows the ion abundances obtained with a conventional rod set at 1.08 mTorr. As the excitation voltage is increased, the precursor ion decreases in intensity $\left(50 \%\right.$ of initial intensity at $\left.69 \mathrm{mV}_{0-p}\right)$, the fragment ions increase and the sum of all ions increases somewhat. At higher excitation amplitudes the fragment ion abundances continue to increase and precursor ions are efficiently converted to fragment ions. Figure $6 \mathrm{~b}$ shows the effects of increasing the excitation time with a conventional rod set. At excitation times up to $500 \mathrm{~ms}$ the fragment ion abundances continue to increase, although the precursor ion is never completely removed.

Figure $6 \mathrm{c}$ and $\mathrm{d}$ show similar plots for the rod set with $4 \%$ octopole with dipole excitation applied between the larger rods. Figure $6 c$ shows that as the excitation amplitude is increased from zero, the precursor ions decrease in abundance $(50 \%$ of initial intensity at $\left.106 \mathrm{mV}_{0-p}\right)$, and the fragment ion intensities increase. At higher excitation amplitudes all precursor ions are converted to fragment ions. Figure $6 \mathrm{~d}$ shows the effect of varying the excitation time between the larger rods. At greater excitation times again the product ion abundances increase. Figure $6 \mathrm{e}$ and $\mathrm{f}$ show the effects of changing the excitation amplitude and excitation time with dipole excitation between the smaller rods. The results are similar to those with excitation between the larger rods. At an excitation amplitude of $96 \mathrm{mV}_{0-p}$ the precursor ions are reduced to $50 \%$ of their initial inten- 

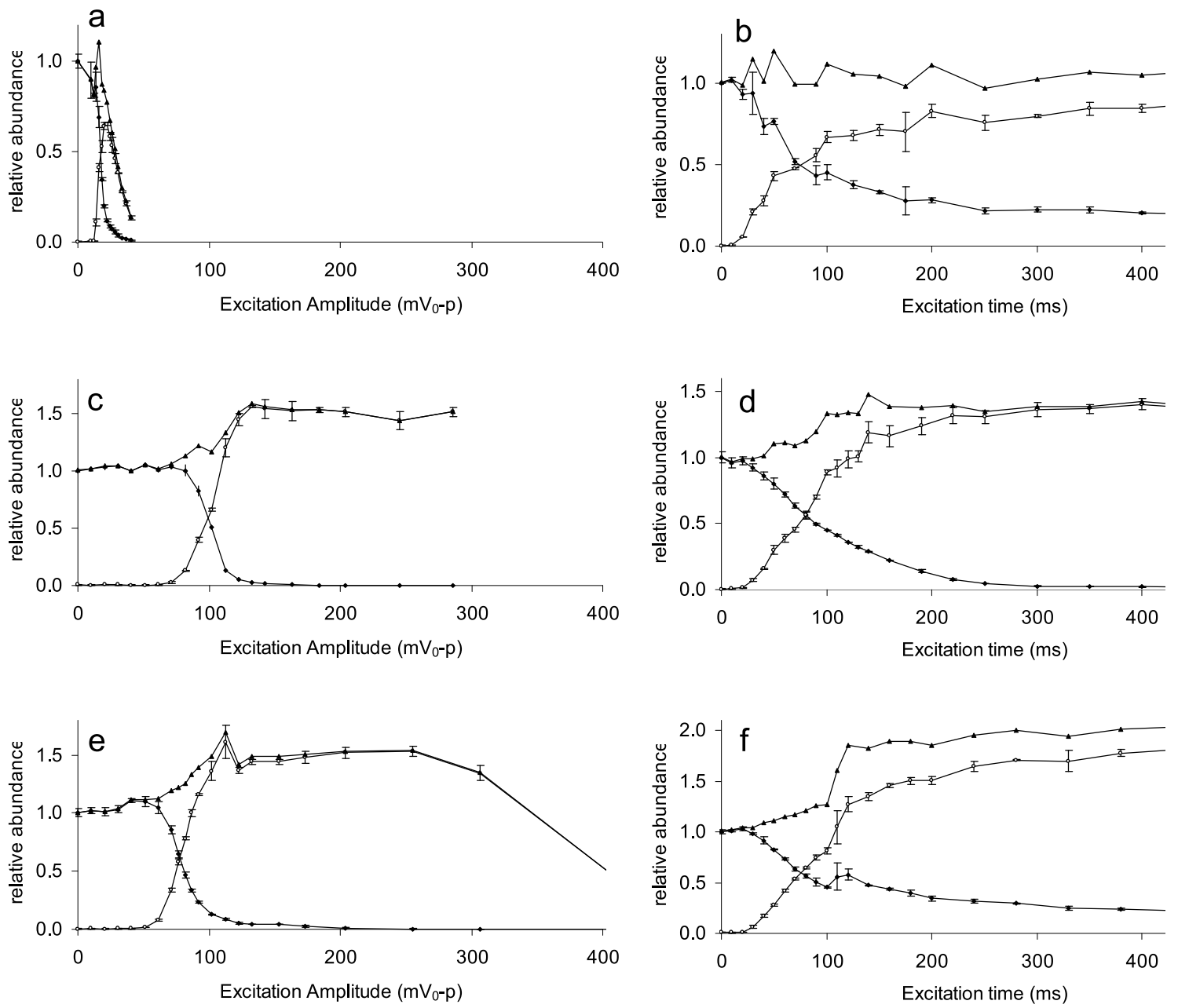

Figure 7. Ion intensities in MS/MS of reserpine at ca. 0.14 mTorr. In each plot the decrease of precursor ions, sum of all fragment ions, and sum of all ions in the spectrum are shown. (a) Conventional rod set, 0.14 mTorr, intensities versus excitation amplitude $(54.2 \mathrm{kHz}),(\mathbf{b})$ conventional rod set, $0.14 \mathrm{mTorr}$, intensities versus excitation time, amplitude $18 \mathrm{mV}_{0-p}(54.2 \mathrm{kHz})$, (c) rod set with $A_{4}=0.04,0.15$ mTorr, excitation between the larger rods, intensities versus excitation amplitude (54.05 $\mathrm{kHz}$ ), (d) rod set with $A_{4}=0.040$, excitation between the larger rods, intensities versus excitation time, amplitude $102 \mathrm{mV}_{0-p}(54.05 \mathrm{kHz})$, (e) rod set with $A_{4}=0.04,0.14 \mathrm{mTorr}$, excitation between the smaller rods, intensities versus excitation amplitude $(57.1 \mathrm{kHz}),(\mathbf{f})$ rod set with $A_{4}=0.04,0.14 \mathrm{mTorr}$, excitation between the smaller rods, intensities versus excitation time, amplitude $81 \mathrm{mV}_{0-p}(57.1 \mathrm{kHz})$.

sity; at higher amplitudes precursors are efficiently converted to product ions. With an amplitude of 96 $\mathrm{mV}_{0-p}$, as the excitation time is increased a greater fraction of precursors is converted to fragments.

The data of Figure 6 show that at a trap pressure of 1.05 mTorr of $\mathrm{N}_{2}$, reserpine ions can be efficiently converted to product ions in every case. The rod sets with added octopole field offer little improvement in MS/MS efficiency over a conventional rod set. A comparison of Figure 6a, c, and e show that with the added octopole field, ions can be excited at greater amplitudes and over a broader range of amplitudes than in a conventional rod set, without loss of ions. With a conventional rod set, the sum of all ions begins to decrease at an amplitude of about $100 \mathrm{mV}_{0-p}$ whereas with the $4 \%$ octopole rod set, the ions can be excited at $300 \mathrm{mV}_{0-p}$ without losses. Because of the smaller field radius, for a given excitation voltage, the conventional rod set produces an electric field about $8 \%$ greater than the rod set with $4 \%$ octopole (Table 2 and eq 26). The differences in excitation voltages that can be applied to the rod set with $4 \%$ octopole field are much greater than this difference.

Figure 7a shows the effect of varying excitation amplitude with a conventional rod set at a pressure of $0.14 \mathrm{mTorr}$. As the amplitude is increased, the precursor ion intensity decreases $(50 \%$ of initial intensity at 18 $\left.\mathrm{mV}_{0-p}\right)$. The fragment ion abundances reach a maximum at about $33 \mathrm{mV}_{0-p}$. Above ca. $20 \mathrm{mV}_{0-p}$ the intensities of all ions decrease. About $60 \%$ of the precursor ions can be converted to product ions. Figure $7 \mathrm{~b}$ shows the effect of increasing the excitation time. Greater excitation times give increased fragmentation, but in contrast to Figure $6 \mathrm{~b}$ the sum of fragment ions remains less than 

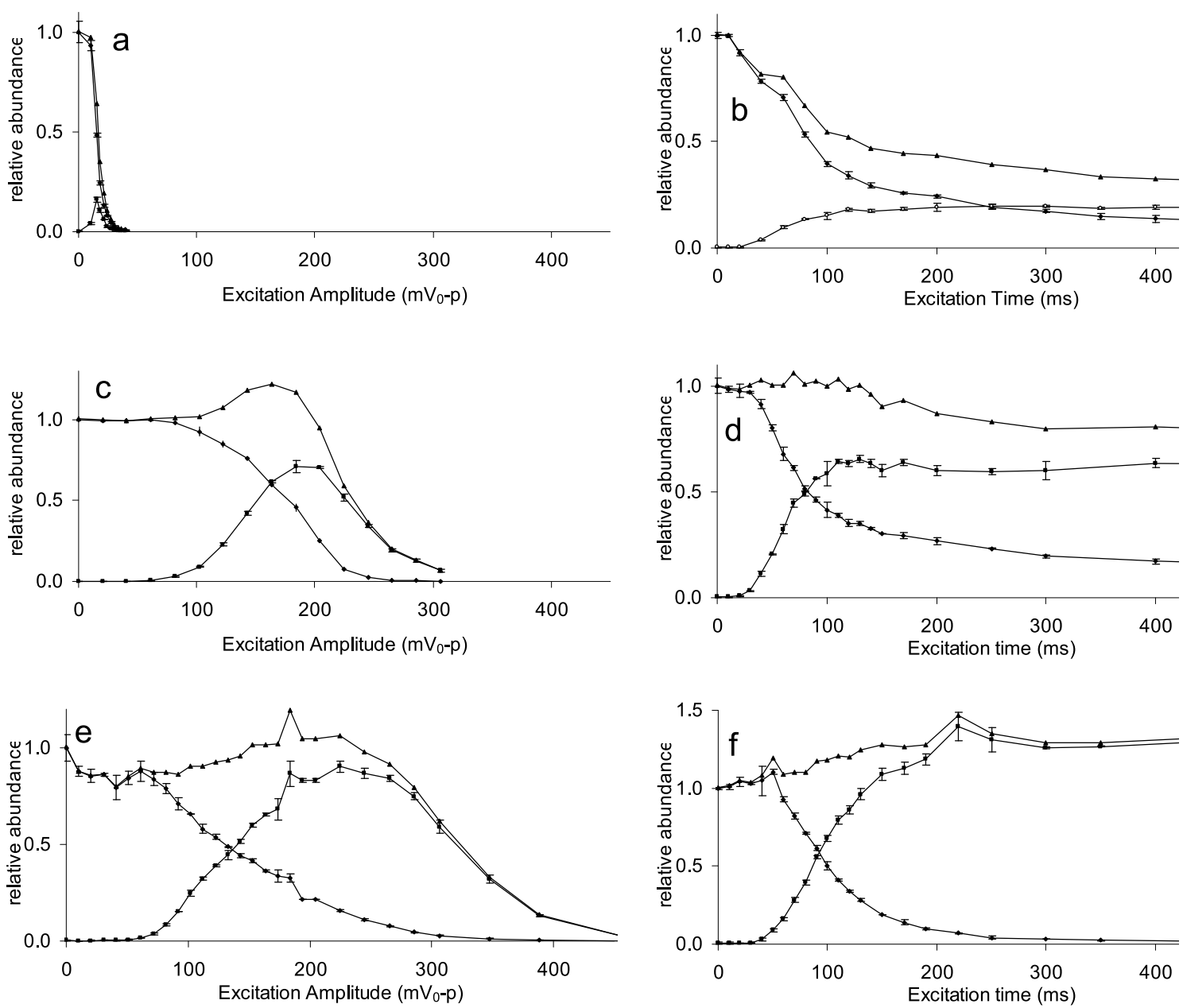

Figure 8. Ion intensities in MS/MS of reserpine at ca. 0.035 mTorr. In each plot the decrease of precursor ions, sum of all fragment ions, and sum of all ions in the spectrum are shown. (a) Conventional rod set, 0.038 mTorr, intensities versus excitation amplitude $(54.2 \mathrm{kHz}),(\mathbf{b})$ conventional rod set, 0.038 mTorr, intensities versus excitation time, amplitude $16 \mathrm{mV}_{O-p}(54.2 \mathrm{kHz})$, (c) rod set with $A_{4}=0.04,0.030 \mathrm{mTorr}$, excitation between the larger rods, intensities versus excitation amplitude (53.3 $\mathrm{kHz}$ ), (d) rod set with $A_{4}=0.040,0.030 \mathrm{mTorr}$, excitation between the larger rods, intensities versus excitation time, amplitude $183 \mathrm{mV}_{0-p}(53.3 \mathrm{kHz}),(\mathbf{e})$ rod set with $A_{4}=0.04,0.035 \mathrm{mTorr}$, excitation between the smaller rods, intensities versus excitation amplitude $(57.1 \mathrm{kHz}),(\mathbf{f})$ rod set with $A_{4}=$ $0.040,0.035 \mathrm{mTorr}$, excitation between the smaller rods, intensities versus excitation time, amplitude $142 \mathrm{mV}_{0-p}(57.1 \mathrm{kHz})$.

the initial precursor ion abundance. Comparison of Figure $7 b$ to Figure $6 a$ and $b$, shows that ions are less efficiently converted to fragment ions. Figure $7 \mathrm{c}$ and $\mathrm{d}$ show data obtained with excitation between the larger rods. Beginning at an amplitude of ca. $80 \mathrm{mV}_{0-p}$ the precursor ion abundance decreases $(50 \%$ of initial intensity at $107 \mathrm{mV}_{0-p}$ ) and the fragment ion abundances increase. At higher amplitudes, precursor ions are efficiently converted to products. Ions can be excited with amplitudes of at least $250 \mathrm{mV}_{0-p}$ without substantial losses. Figure $7 \mathrm{~d}$ shows the effect of increasing the excitation time. Above $100 \mathrm{~ms}$, fragment ion abundances continue to increase and all precursor ions can be converted to fragment ions. Figure $7 \mathrm{e}$ and $\mathrm{f}$ show similar experiments with excitation between the smaller rods. At an excitation amplitude of about $81 \mathrm{mV}_{0-p}$ the precursor is reduced to $50 \%$ of its initial intensity (Figure 7e) and above this amplitude, the intensities of fragment ions increase and all precursors can be converted to fragments. Figure $7 \mathrm{f}$ shows that precursor ions can be converted to fragment ions with high efficiencies at excitation times greater than about $250 \mathrm{~ms}$. Overall the data in Figure 7 again show that ions can be excited with substantially greater amplitudes and over a greater range of amplitudes in the rod set with added octopole field without loss of ions (i.e., the sum of all ions remains approximately constant). Further, the data of Figure 7 show that the rod set with $4 \%$ octopole field has somewhat higher MS/MS efficiency under these operating conditions.

Figure 8a shows that, with a conventional rod set at a pressure of $0.038 \mathrm{mTorr}$, increasing the amplitude 
Table 3a. MS/MS efficiencies (\%) at ca. 1 mTorr

\begin{tabular}{|c|c|c|c|}
\hline Excitation time & $\begin{array}{c}\text { Conventional rod set } \\
\text { (1.14 mTorr) }\end{array}$ & $\begin{array}{l}\text { Larger rods } \\
(1.09 \mathrm{mTorr})\end{array}$ & $\begin{array}{l}\text { Smaller rods } \\
\text { (1.04 mTorr) }\end{array}$ \\
\hline $50 \mathrm{~ms}$ & $41\left(63 \mathrm{mV}_{o-p}\right)$ & $98\left(115 \mathrm{mV}_{o-p}\right)$ & $93\left(100 \mathrm{mV}_{o-p}\right)$ \\
\hline $100 \mathrm{~ms}$ & $45\left(60 \mathrm{mV}_{o-p}\right)$ & $99\left(105 \mathrm{mV}_{o-p}\right)$ & $99\left(90 \mathrm{mV}_{o-p}\right)$ \\
\hline 30,000 cycles & $77\left(53 \mathrm{mV}_{o-p}, 482 \mathrm{~ms}\right)$ & $157\left(90 \mathrm{mV}_{o-p}, 484 \mathrm{~ms}\right)$ & $144\left(77 \mathrm{mV}_{o-p} 457 \mathrm{~ms}\right)$ \\
\hline
\end{tabular}

from zero causes the precursor ion intensity to decrease $\left(50 \%\right.$ of initial intensity at $\left.16 \mathrm{mV}_{0-p}\right)$, with the formation of product ions of low abundance (maximum intensity at about $\left.14 \mathrm{mV}_{0-p}\right)$. At higher amplitudes, all ion intensities decrease and, in comparison to experiments at higher pressures (Figures 6a and 7a) the efficiency of converting precursor ions to product ions is poor. Figure $8 \mathrm{~b}$ shows that with a conventional rod set, the abundance of fragment ions increases slightly at excitation times above $100 \mathrm{~ms}$, but remains low. Figure 8c and $\mathrm{d}$ show data obtained with excitation applied between the larger rods. Figure 8c shows that as the excitation amplitude is increased from zero, the precursor ion intensity decreases $\left(50 \%\right.$ of initial intensity at $\left.183 \mathrm{mV}_{0-p}\right)$ and the fragment ion abundances increase, with equal intensities at about $150 \mathrm{mV}_{0-p}$. The fragments reach maximum intensity at ca. $200 \mathrm{mV}_{0-p}$. At higher amplitudes, the intensities of all ions decrease. Nevertheless about $75 \%$ of the precursor ions can be converted to fragment ions. Figure $8 \mathrm{~d}$ shows the effect of increasing excitation time between the large rods. At times greater than about $100 \mathrm{~ms}$ the abundance of fragments increases to ca. $70 \%$ of the initial precursor intensity. Figure $8 \mathrm{e}$ and $\mathrm{f}$ show the results of similar experiments with excitation between the smaller rods. Figure 8e shows that as the amplitude increases the precursor ion abundance decreases $(50 \%$ of initial intensity at 142 $\left.m V_{O-p}\right)$ and the fragment ion abundances increase. The fragment ion abundances continue to increase until at around $220 \mathrm{mV}_{0-p}$, the intensities of all ions begin to decrease. Figure $8 \mathrm{f}$ shows the effect of increasing the excitation time. Above $100 \mathrm{~ms}$ the abundance of fragment ions continues to increase and nearly all precursor ions can be converted to fragment ions.

Comparison of Figures 6,7 , and 8 shows that as the pressure decreases below $1 \mathrm{mTorr}$, the rod set with $4 \%$ octopole field shows greater MS/MS efficiencies than a conventional rod set. This is most apparent in Figure 8 where there are dramatic differences between the rod sets. At this pressure, with a conventional rod set, only about $15 \%$ (Figure 8 a) to $20 \%$ (Figure $8 b$ ) of the precursor ions are converted to fragment ions. With the rod set with $4 \%$ octopole field, and with excitation between the larger rods about $70 \%$ of the initial precursor intensity appears as fragments. Figure $8 \mathrm{e}$ and $\mathrm{f}$ show that with excitation between the smaller rods, nearly all the precursor ions can be converted to fragment ions. Under these conditions, the MS/MS efficiency with the rod set with a $4 \%$ added octopole field is about five times greater than a conventional rod set. In addition, when a $4 \%$ octopole field is added, ions can be excited with a broader range of amplitudes without loss of ions.

The MS/MS efficiency is expected to be compound dependent. Thus the experiments with reserpine ions were repeated with protonated ions of morphine-3- $\beta$ D-glucuronide (a metabolite of morphine). Results similar to those with reserpine ions were obtained (data not shown). When ions of morphine-3- $\beta$-D-glucuronide dissociate, they produce a fragment at $m / z=286$. This fragment ion was found to be more difficult to dissociate than its precursor molecular ion or ions of reserpine. For MS/MS experiments, the $\mathrm{m} / \mathrm{z} 286$ fragment was produced by increasing the voltage difference between the orifice and skimmer from 170 to $220 \mathrm{~V}$, to give the highest abundance of the $\mathrm{m} / \mathrm{z} 286$ ion. The $\mathrm{m} / \mathrm{z} 286$ fragment ions were then isolated in the linear trap, trapped at $q=0.23$ and excited for MS/MS at a variety of excitation times. Excitation amplitudes were chosen to give 95 to $100 \%$ depletion of the $\mathrm{m} / \mathrm{z} 286$ ion. Table $3 a$ shows the MS/MS efficiencies (summed intensities of fragments divided by initial precursor ion intensity $\times$ $100 \%$ ) obtained at a pressure of ca. 1 mTorr with a conventional rod set and a rod set with $4 \%$ octopole field. Excitation times of 50, 100, and about $480 \mathrm{~ms}$ were tested. The latter corresponds to 30,000 cycles of the excitation voltage, the longest time possible with the waveform generator used. At $1 \mathrm{mTorr}$, the rod sets with $4 \%$ octopole field showed MS/MS efficiencies greater by about a factor of two. The rod sets were then tested at a pressure of ca. 0.05 mTorr, with the results shown in Table 3. With the conventional rod set, almost no fragment ions could be formed at any excitation time. However with the $4 \%$ octopole rods, some fragments could be formed with 100 ms excitation time and with

Table 3b. MS/MS efficiencies (\%) at ca. $0.05 \mathrm{mTorr}$

\begin{tabular}{|c|c|c|c|}
\hline Excitation time & $\begin{array}{c}\text { Conventional rod set } \\
(0.053 \mathrm{mTorr})\end{array}$ & $\begin{array}{l}\text { Larger rods } \\
(0.046 \mathrm{mTorr})\end{array}$ & $\begin{array}{l}\text { Smaller rods } \\
(0.048 \text { mTorr })\end{array}$ \\
\hline $50 \mathrm{~ms}$ & $0.0\left(18 \mathrm{mV}_{o-p}\right)$ & $0.1\left(135 \mathrm{mV}_{o-p}\right)$ & $0\left(128 \mathrm{mV}_{o-p}\right)$ \\
\hline $100 \mathrm{~ms}$ & $0.1\left(19 \mathrm{mV}_{o-p}\right)$ & $3\left(120 \mathrm{mV}_{o-p}\right)$ & $17\left(115 \mathrm{mV}_{o-p}\right)$ \\
\hline 30,000 cycles & $0.1\left(20 \mathrm{mV}_{o-p}, 479 \mathrm{~ms}\right)$ & $96\left(90 \mathrm{mV}_{o-p} 487 \mathrm{~ms}\right)$ & $106\left(80 \mathrm{mV}_{o-p} 452 \mathrm{~ms}\right)$ \\
\hline
\end{tabular}



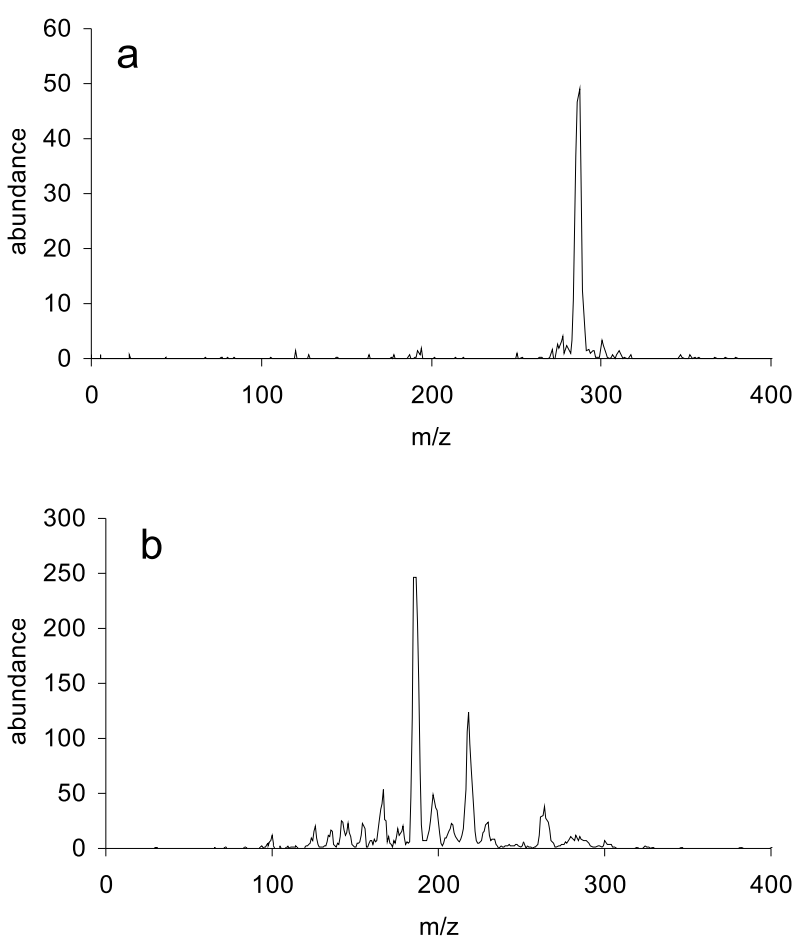

Figure 9. MS/MS spectra of the $m / z 286$ fragment of morphine3 - $\beta$-D-glucuronide with (a) a conventional rod set, 0.053 mTorr, $q$ $=0.23$, excitation of $20 \mathrm{mV}_{0-p}$ at $62.63 \mathrm{kHz}$ applied for $479 \mathrm{~ms}$, (b) rod set with $A_{4}=0.040,0.048 \mathrm{mTorr}$, excitation of $80 \mathrm{mV}_{0-p}$ at $66.37 \mathrm{kHz}$ applied for $452 \mathrm{~ms}$ between the smaller rods.

excitation for $480 \mathrm{~ms}$ the MS/MS efficiency increases to about $100 \%$. The differences in fragment ion spectra are dramatic. Figure 9a show the MS/MS spectrum obtained with a conventional rod set. Although there is 93\% depletion of the precursor ion, almost no fragment ions are formed. Figure $9 \mathrm{~b}$ shows the MS/MS spectrum with excitation between the smaller rods of the $4 \%$ octopole rod set. There is $97 \%$ depletion of the precursor ion, and abundant fragment ions are formed. The MS/MS efficiency is calculated to be $106 \%$.

The data in Table 3 illustrate another effect. At a pressure of $1 \mathrm{mTorr}$, and with the rod set with $4 \%$ octopole field, 100\% MS/MS efficiency was possible with a $50 \mathrm{~ms}$ excitation time. To get a similar MS/MS efficiency $(77 \%)$ with the conventional rod set, an excitation time of $482 \mathrm{~ms}$ was required, nearly ten times longer. Thus, under some operating conditions, it seems likely that with the rod set with added octopole field, ions can be dissociated with higher excitation amplitudes and shorter excitation times to improve the duty cycle of an MS/MS experiment.

The higher MS/MS efficiency with an added multipole field has been attributed to the frequency shift that occurs as the amplitude of oscillation increases [1a, 8]. With a pure quadrupole field, the frequency of oscillation is independent of amplitude. If the amplitude of excitation is increased in an attempt to gain a greater degree of fragmentation through more energetic collisions, ions can be ejected. This is illustrated in Figure 10.
Figure 10a shows the trajectory of an ion of $m / z=609$, trapped at $q=0.20$, excited at its resonant frequency of $54.739 \mathrm{kHz}$, in the presence of 0.14 mTorr of $\mathrm{N}_{2}$ (trapping frequency $768 \mathrm{kHz}$ ). The collision cross section was taken as $280 \AA^{2}$ [24], which gives a damping constant $\lambda=187 \mathrm{~s}^{-1}$. The excitation amplitude is 10 $\mathrm{mV}_{0-p}$. These conditions are chosen to match approximately the conditions in Figure 7a where the precursor starts to show depletion. The amplitude of oscillation increases with time until a steady state is reached where the amplitude is ca. $0.8 r_{0}$ or $3.3 \mathrm{~mm}$. Figure 10b shows the trajectory when the excitation amplitude is increased to $35 \mathrm{mV}_{0-p}$. The amplitude of oscillation increases more rapidly and after about $2000 \mathrm{rf}$ cycles $(2.6 \mathrm{~ms})$ the ion strikes a rod and is lost. This is qualitatively similar to the data of Figure 7a where ions are lost at amplitudes greater than about $30 \mathrm{mV}_{0-p}$. In contrast, when there is an octopole field added, the ion trajectories are quite different. Figure 11a shows excitation of an ion of $\mathrm{m} / \mathrm{z}=609$ in quadrupole field $\left(r_{0}=4.5\right.$ $\mathrm{mm}$ ) with a $+4 \%$ octopole component, at a pressure of 0.14 mTorr $(q=0.2031$, trapping frequency $768 \mathrm{kHz}$; the conditions of Figure 7e). The excitation amplitude is 75
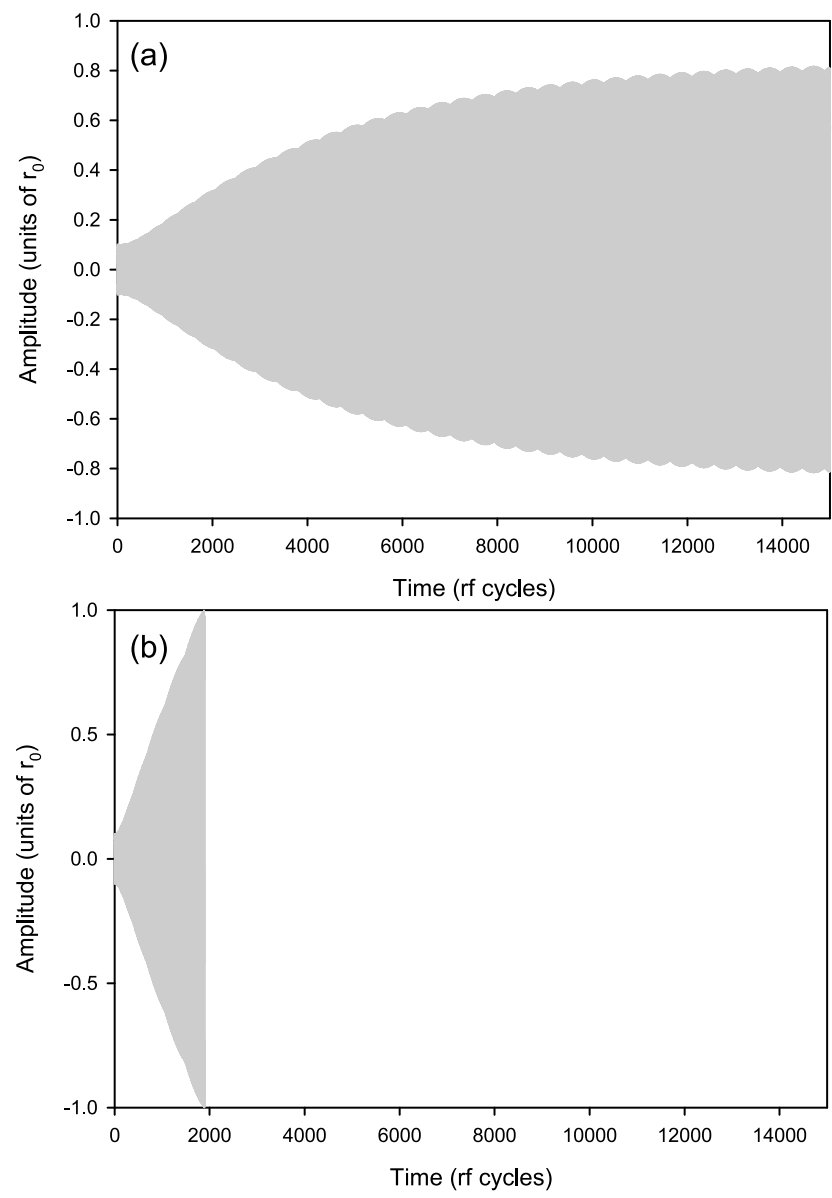

Figure 10. Ion trajectory in an ideal quadrupole field, with dipole excitation, $m / z=609, q=0.20,0.14 \mathrm{mTorr}$ of $\mathrm{N}_{2}$, trapping rf frequency $768 \mathrm{kHz}$, excitation frequency $54.739 \mathrm{kHz}, r_{0}=4.17$ $\mathrm{mm}$, (a) excitation amplitude $10 \mathrm{mV}_{0-p}$ (b) excitation amplitude 35 $\mathrm{mV}_{0-p}$. 

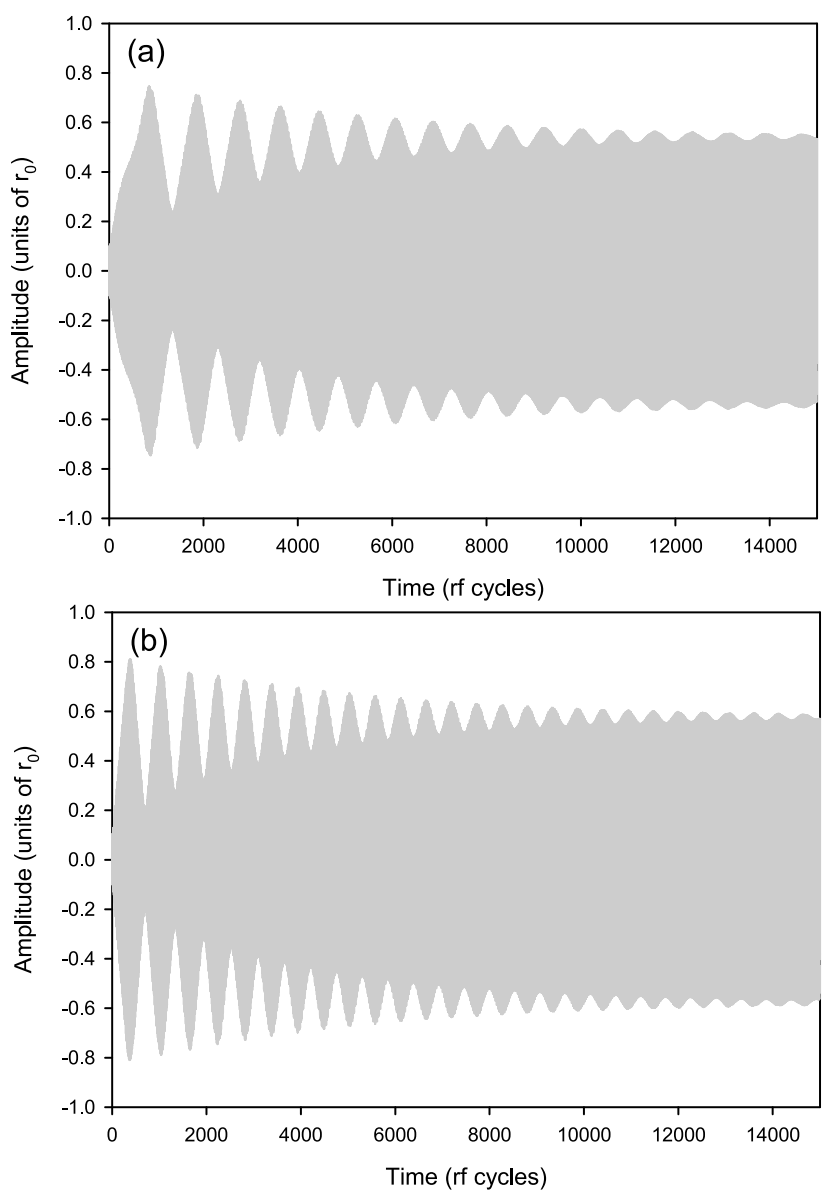

Figure 11. Ion trajectory in the $x$ direction in a rod set with $A_{4}=$ $+0.04, q=0.2031,0.14$ mTorr, trapping rf frequency $768 \mathrm{kHz}$, excitation frequency $56.954 \mathrm{kHz}$. (a) Excitation amplitude 75 $\mathrm{mV}_{O-p},\left(\right.$ b) excitation amplitude $150 \mathrm{mV}_{O-p}$.

$\mathrm{mV}_{0-p}$. The ions are excited at $56.954 \mathrm{kHz}$, which is 1.351 $\mathrm{kHz}$ above their unperturbed frequency $\frac{\omega_{0}}{2 \pi}=$ $55.602 \mathrm{kHz}$. The amplitude of ion oscillation increases but a beat-like pattern is formed. Because the frequency of ion oscillation depends on the amplitude of oscillation, the excitation frequency is alternately in and out of phase with the excitation. Damping by collisions leads to a steady state where the amplitude of oscillation is about $0.54 r_{0}$ or $2.4 \mathrm{~mm}$. The two amplitudes calculated from eq 16 are $1.8 \mathrm{~mm}$ and $2.2 \mathrm{~mm}$, in reasonable agreement. When the excitation amplitude is doubled to $150 \mathrm{mV}_{0-p}$ the trajectory of Figure $11 \mathrm{~b}$ is calculated. The amplitude of oscillation is greater which means there are greater frequency shifts. A transient beat-like pattern is formed before a steady state is reached with an oscillation amplitude of about $0.65 r_{0}(2.9 \mathrm{~mm})$. The beat-like pattern formed when there was an added octopole or hexapole field was described by Franzen et al. [1a], but the effects of damping by collisions were not discussed or illustrated. Figure 11 shows that when there is an added octopole field, ions can be excited with substantially higher amplitudes without striking the rods, as is seen in the experimental data of Figures 6,7 , and 8 .

Collings et al. [7] have described in detail resonant excitation in a low pressure (nominally $3 \times 10^{-5}$ torr) linear trap constructed with a round rod set that has essentially the same geometry as our conventional rod set. In contrast to our results at a similar pressure, reserpine ions could be efficiently fragmented. The reasons for the different behavior are not clear. The more efficient fragmentation in that experiment may be due to a number of effects, such as a locally higher gas density especially near the trap entrance, higher internal energies of ions entering the trap, or operation of the trap at $816 \mathrm{kHz}$ instead of $768 \mathrm{kHz}$. The higher frequency and slightly higher trapping $q$ in that experiment $(q=0.21$ versus $q=0.20$ ) gives a somewhat higher effective potential well depth of $8.0 \mathrm{~V}$ compared to a well depth of $6.4 \mathrm{~V}$ in our experiments. (Our experiments with the $4 \%$ octopole rod set had a well depth of 7.6 V.) Based on trajectory calculations, Collings et al. attributed the efficient fragmentation of ions at the low pressure to the presence of higher multipoles in the potential, arising from the use of round rods. A beat pattern more complex than that of Figure 11 was observed in trajectory calculations.

\section{Conclusions}

The effective potential model for ion motion in the linear trap gives an anharmonic oscillator with equal but opposite sign frequency shifts for ion motion between the $x$ and $y$ rods, and predicts bistable behavior for excitation amplitudes above a critical value. Experiments show these frequency shifts and also show bistable behavior for excitation between the larger rods but not the smaller rods. A comparison of MS/MS efficiencies between a conventional rod set and a rod set with $4 \%$ added octopole field shows that both give high MS/MS efficiencies at a trap pressure of 1.05 mTorr of $\mathrm{N}_{2}$, but as the pressure is decreased the efficiency of the rod set with added octopole field increases over that of the conventional rod set. This is attributed to a shift of oscillation frequency with increased oscillation amplitude which favors ion fragmentation over ion ejection. This work and the work of Collings et al. [8] show that the efficiency of fragmentation in a linear trap can be improved by the addition of higher multipoles to the potential. Other effects, such as frequency shifts and asymmetric excitation profiles offer considerable scope for further theoretical and experimental investigation.

\section{Acknowledgments}

This work was supported by the Natural Sciences and Engineering Research Council and SCIEX Division of MDS Inc. The authors thank Michael Sudakov for the programs that calculate multipole potentials in round rod sets and ion trajectories. 


\section{References}

1. (a) Franzen, J.; Gabling, R.-H.; Schubert, M.; Wang, Y.; Nonlinear Ion Traps. In Practical Aspects of Ion Trap Mass Spectrometry, Vol I; March, R. E.; Todd, J. F. J., Eds.; CRC Press: Boca Raton, FL, 1995; 49-167. (b) Wang, Y.; Franzen, J. The nonlinear QUISTOR. Part 1. Potential distribution in hyperboloidal QUISTORS. Int. J. Mass Spectrom. Ion Processes 1992, 112, 167-178. (c) Wang, Y.; Franzen, J.; Wanczek, K. P. The nonlinear resonance ion trap. Part 2. A general theoretical analysis. Int. J. Mass Spectrom. Ion Processes 1993, 124, 125-144. (d) Wang, Y.; Franzen, J. The nonlinear ion trap. Part 3. Multipole components in three types of practical ion traps. Int. J. Mass Spectrom. Ion Processes 1994, 132, 155-172. (e) Franzen, J. The nonlinear ion trap. Part 4 . Mass selective instability scan with multipole superposition. Int. J. Mass Spectrom. Ion Processes 1993, 125, 165-170. (f) Franzen, J. The nonlinear ion trap. Part 5. Nature of nonlinear resonances and resonant ejection. Int. J. Mass Spectrom. Ion Processses 1994, 130, 15-40. (g) Franzen, J. Simulation study of an ion cage with superimposed multipole fields. Int. J. Mass Spectrom. Ion Processes 1991, 106, 63-78.

2. Sudakov, M. Effective potential and the ion axial beat motion near the boundary of the first stable region in a nonlinear ion trap. Int. J. Mass Spectrom. 2001, 206, 27-43.

3. Cai, Y; Peng, W.-P.; Kuo, S.-J.; Chang, H.-C. Calibration of an audio-frequency ion trap mass spectrometer. Int. J. Mass Spectrom. 2002, 214, 63-73.

4. Schwartz, J. C.; Senko, M. W.; Syka, J. E. P. A two-dimensional quadrupole ion trap mass spectrometer. J. Am. Soc. Mass Spectrom. 2002, 13, 659-669.

5. Hager, J. W. A new linear ion trap mass spectrometer. Rapid Commun. Mass Spectrom. 2002, 16, 512-526.

6. Douglas, D. J.; Frank, A. J.; Mao, D. Linear ion traps in mass spectrometry. Mass Spectrom. Rev. 2004, 24, 1-29.

7. Collings, B. A.; Stott, W. R.; Londry, F. A. Resonant excitation in a low pressure linear ion trap. J. Am. Soc. Mass Spectrom. 2003, 14, 622-634.

8. (a) Sudakov, M.; Douglas, D. J. Linear quadrupoles with added octopole fields. Rapid Commun. Mass Spectrom. 2003, 17, 2290-2294. (b) Ding, C.; Konenkov, N. V.; Douglas, D. J. Quadrupole mass filters with octopole fields. Rapid Commun. Mass Spectrom. 2003, 17, 2495-2502.

9. Landau, L. D.; Lifshitz, E. M. Mechanics, 3rd ed.; Pergamon Press: New York, NY, 1960; pp 74-93.

10. Gerlich, D. Advances in Chemical Physics; Vol LXXXII. Inhomogeneous RF fields: A versatile tool for the study of processes with slow ions; John Wiley and Sons: New York, NY, 1992; pp 1-176.

11. Campbell, J. M.; Collings, B. A.; Douglas, D. J. A linear ion trap time-of-flight system with tandem mass spectrometry capabilities. Rapid Commun. Mass Spectrom. 1998, 12, 1463-1474.

12. Collings, B. A.; Campbell, J. M.; Mao, D.; Douglas, D. J. A combined linear ion trap time-of-flight system with improved performance and $\mathrm{MS}^{\mathrm{n}}$ capabilities. Rapid Commun. Mass Spectrom. 2001, 15, 1777-1795.
13. Todd, J. F. J.; Lawson, G.; Bonner, R. F. Quadrupole Ion Traps. In Quadrupole Mass Spectrometry and its Applications, Dawson, P. H., Ed.; AIP Press: Woodbury, NY, 1995, pp 210-214.

14. Sevugarajan, S.; Menon, A. G. Field imperfection induced axial secular frequency shifts in nonlinear ion traps. Int. J. Mass Spectrom. 1999, 189, 53-61.

15. Sudakov, M.; Kumashiro, S. Theory and simulations of resonance excitation of ion vibrations in nonlinear and pure quadrupole ion traps. Part 1; Proceedings of the 51st ASMS Conference on Mass Spectrometry and Allied Topics; Montreal, Canada, June, 2003; pp 8-12.

16. March, R. E.; Weir, M. R.; Tkaczyk, M.; Londry, F. A.; Alfred, R. L.; Franklin, A. M.; Todd, J. F. J. Kinetic energy effects in an ion ensemble subjected to mass-selective isolation and resonance excitation: A simulation study. Org. Mass Spectrom. 1993, 28, 499-509.

17. Williams, J. D.; Cox, K. A.; Cooks, R. G.; McLuckey, S. A.; Hart, K. J.; Goeringer, D. E. Resonance ejection ion trap mass spectrometry and nonlinear field contributions: The effect of scan direction on mass resolution. Anal. Chem. 1994, 66, 725-729.

18. Splendore M.; Londry, F. A.; March R. E.; Morrison, R. J. S.; Perrier, P.; Andre, J. A. Simulation study of ion kinetic energies during resonant excitation in a stretched ion trap. Int. J. Mass Spectrom. Ion Processes 1996, 156, 11-29.

19. (a) Alheit, R.; Chu, X. Z.; Hoefer, M.; Holzki, M.; Werth, G. Nonlinear collective oscillations of an ion cloud in a Paul trap. Phys. Rev. A 1997, 56, 4023-4031. (b) Chu, X. Z.; Holzki, M.; Alheit, R.; Werth, G. Observation of high-order motional resonances of an ion cloud in a Paul trap. Int. J. Mass Spectrom. Ion Processes 1998, 173, 107-112.

20. Nappi, M.; Frankevich V.; Soni, M.; Cooks, R. G. Characteristics of a broad band Fourier transform ion trap mass spectrometer. Int. J. Mass Spectrom. 1998, 177, 91-104.

21. Bologoliubov, N. N.; Mitropolsky, Y. A. Asymptotic methods in the theory of noninear oscillations; Hindustan Publishing Corporation: Delhi, India, 1961; p 245

22. Makarov, A. A. Resonance ejection from the Paul trap: A theoretical treatment incorporating a weak octapole field. Anal. Chem. 1996, 68, 4257-4263.

23. Chen, Y.-L.; Collings, B. A.; Douglas, D. J. Collision cross sections of myoglobin and cytochrome $c$ ions with $\mathrm{Ne}, \mathrm{Ar}$, and Kr. J. Am. Soc. Mass Spectrom. 1997, 8, 681-687.

24. Javahery, G.; Thomson, B. A. A segmented radiofrequencyonly quadrupole collision cell for measurements of ion collision cross section on a triple quadrupole mass spectrometer. J. Am. Soc. Mass Spectrom. 1997, 8, 697-702.

25. Douglas, D. J.; Glebova, T. A.; Konenkov, N. V.; Sudakov, M. Y. Spatial harmonics of the field in a quadrupole mass filter with circular electrodes. Tech. Phys. 1999, 44, 1215-1219.

26. Ding, L.; Sudakov, M.; Brancia F. L.; Giles, R.; Kumashiro, S. A digital ion trap mass spectrometer coupled with atmospheric pressure ion sources. J. Mass Spectrom. 2004, 39, 471-484. 